\title{
ON A LUSIN THEOREM FOR CAPACITIES
}

\author{
JOHANNES WIESEL
}

\begin{abstract}
Let $X$ be a compact metric space and let $v$ be a sub-additive capacity defined on $X$. We show that Lusin's theorem with respect to $v$ holds if and only if $v$ is continuous from above.
\end{abstract}

\section{INTRODUCTION}

Throughout this article, we work on a compact metric space $(X, d)$. Let us quickly recall the classical Lusin theorem, which reads as follows:

Theorem 1.1 (Lusin, 6]). Let $\mu$ be a (Borel) probability measure on $X$ and fix $\varepsilon>0$. Then for every Borel-measurable function $u: X \rightarrow \mathbb{R}$ there exists a compact set $K=K(u, \varepsilon)$ such that $\mu(X \backslash K) \leq \varepsilon$ and the restriction $\left.u\right|_{K}$ is continuous.

In recent years, several articles have identified sufficient conditions for replacing the measure $\mu$ by a class of capacities or sublinear expectations: see e.g. 11 for an extension to Dempster capacities, 3, 5, for a Lusin theorem with respect to fuzzy measures, as well as 7 for a result concerning g-Expectations.

The aim of this article is to unify these recent advances by identifying a necessary and sufficient condition for an extension of Lusin's theorem to the class of subadditive capacities. To the best of our knowledge this is the first result of its kind. Our main result can be stated as follows:

Theorem 1.2. Let $(X, d)$ be a compact metric space and let $v$ be a sub-additive capacity. Then the property

for all $\left(O_{n}\right)_{n \in \mathbb{N}}, O_{n}$ open, $O_{n} \downarrow O \quad \Rightarrow \quad \lim _{n \rightarrow \infty} v\left(O_{n}\right)=v(O)$

holds if and only if the property

for all $\varepsilon>0$ and for all Borel measurable functions $u: X \rightarrow \mathbb{R}$ there

exists a compact set $K=K(u, \varepsilon) \subseteq X$, such that $v(X \backslash K) \leq \varepsilon$ and $\left.u\right|_{K}$ is continuous

is satisfied.

Remark 1.3. As any probability measure $\mu$ is a subadditive capacity, Theorem 1.2 implies Theorem 1.1.

In conclusion, Lusin's theorem can only hold for a class of "very regular" capacities. This class comprises e.g. the supremum of a finite number of probability measures, but not the supremum of an infinite number of probability measures in general:

Date: June 17, 2021.

Key words and phrases. Capacity, non-additive measure, Lusin's theorem, continuity.

MSC 2010 Classification: 60J45, 28A12, 28E10

We thank Massimo Marinacci for helpful comments. 
indeed, classical examples of 2-alternating capacities, which are e.g. given in [4] and are motivated by distributional robustness, do not typically satisfy the above continuity condition as we will discuss in Section 3

The remainder of the article is structured as follows: In Section 2 we recall basic notions in the theory of capacities and set up the notation used throughout the article. We then provide a counterexample to the main result of 1 in Section 3 motivating the quest for stricter regularity conditions on the class of sub-additive capacities. In Section 4 we recall and discuss our main result, before we provide its proof in Section 5 .

\section{Notation}

Definition 2.1. Let $(X, d)$ be a metric space and let $\mathcal{B}$ denote its Borel $\sigma$-algebra.

- A capacity $v: \mathcal{B} \rightarrow \mathbb{R}$ is a set function satisfying

(1) $v(\emptyset)=0, \quad v(X)=1$,

(2) $A \subseteq B \Rightarrow v(A) \leq v(B)$,

(3) $A_{n} \uparrow A \Rightarrow \lim _{n \rightarrow \infty} v\left(A_{n}\right)=v(A)$,

- A Choquet capacity fulfils (1)-(3) and

$$
F_{n} \text { closed, } F_{n} \downarrow F \Rightarrow \lim _{n \rightarrow \infty} v\left(F_{n}\right)=v(F) .
$$

- A Dempster capacity fulfils (1)-(3) and (C) for all sequences of sets $\left(A_{n}\right)_{n \in \mathbb{N}}$ such that $A_{n} \supseteq \bar{A}_{n+1}$ for all $n \in \mathbb{N}$.

Definition 2.2. A capacity $v$ is called sub-additive, if $v(A \cup B) \leq v(A)+v(B)$ for all Borel sets $A, B$.

Definition 2.3. A capacity $v$ is called 2-alternating or submodular, if

$$
v(A \cup B)+v(A \cap B) \leq v(A)+v(B)
$$

for all Borel sets $A, B$.

Definition 2.4. We define the core of a capacity $v$ as

$$
\operatorname{core}(v):=\{\nu \in \mathcal{P}(X): \nu(A) \leq v(A) \text { for all } A \in \mathcal{B}\},
$$

where $\mathcal{P}(X)$ denotes the set of Borel-probability measures on $X$.

For a Borel set $A$ and $\delta>0$ we define its open $\delta$-neighbourhood as

$$
A^{\delta}:=\inf \left\{x \in X: \inf _{y \in A} d(x, y)<\delta\right\}
$$

and its closed $\delta$-neighbourhood as

$$
A^{\bar{\delta}}:=\inf \left\{x \in X: \inf _{y \in A} d(x, y) \leq \delta\right\} .
$$

We also denote the closure of a set $A \in \mathcal{B}$ by $\bar{A}$. 


\section{A counterexample to 1}

The main result of [1] is the following extension of Lusin's theorem to 2-alternating Dempster capacities:

Proposition 3.1. Let $v$ be a 2-alternating Dempster capacity defined on $\mathcal{B}$. Suppose that $u: X \rightarrow \mathbb{R}$ is a Borel measurable function. Given $\varepsilon>0$ there exists a compact set $K$ such that $v(X \backslash K) \leq \varepsilon$ and $\left.u\right|_{K}$ is continuous.

Let us now consider the following example:

Example 3.2 (see [4, Example 5]). Let $\varepsilon, \delta>0, X$ be a compact metric space and fix a probability measure $\mu$ on $X$. Then according to [4, Example 5], $v$ defined by

$$
v(A)=\min \left(\mu\left(A^{\bar{\delta}}\right)+\varepsilon, 1\right)
$$

for all closed sets $A \neq \emptyset$ is a 2-alternating capacity. We recall here that

$$
A^{\bar{\delta}}:=\inf \left\{x \in X \quad: \inf _{y \in A} d(x, y) \leq \delta\right\}
$$

is the closed $\delta$-neighbourhood of $A$. Furthermore

$$
\operatorname{core}(v)=\left\{\nu \in \mathcal{P}(X): \nu(A) \leq \mu\left(A^{\bar{\delta}}\right)+\varepsilon \text { for all } A \in \mathcal{B}\right\} .
$$

We check that it is also a Dempster capacity: take any sets $A_{n}$ such that $A_{n} \supseteq$ $\bar{A}_{n+1}$. Let $A_{n} \downarrow A$. As $A_{n} \supseteq \bar{A}_{n+1} \supseteq A_{n+1}$ this implies that $\bar{A}_{n} \downarrow A$, and as $X$ is compact, in particular $A$ is compact. Furthermore $\bar{A}_{n}^{\bar{\delta}}=A_{n}^{\bar{\delta}}$ and thus

$$
\left(\bigcap_{n \in \mathbb{N}} \bar{A}_{n}\right)^{\bar{\delta}}=\bigcap_{n \in \mathbb{N}} A_{n}^{\bar{\delta}}
$$

Indeed, the $\subseteq$-relation is clear. Now take $y \in X$ such that for all $n \in \mathbb{N}$ there exists some $x_{n} \in \bar{A}_{n}$ with $d\left(x_{n}, y\right) \leq \delta$. As $\bar{A}_{n}$ is compact, there either exists $x \in \bigcap \bar{A}_{n}$ and a subsequence of $x_{n}$ converging to $x$, or $A_{n}=\emptyset$ for all large $n \in \mathbb{N}$. In the second case the claim is trivial. In the first case, in particular $d(y, x) \leq$ $\limsup _{n \rightarrow \infty} d\left(y, x_{n}\right) \leq \delta$, so $y \in\left(\bigcap_{n \in \mathbb{N}} \bar{A}_{n}\right)^{\bar{\delta}}$. This shows (1). We now conclude that

$$
\mu\left(A^{\bar{\delta}}\right)=\mu\left(\left(\bigcap_{n \in \mathbb{N}} \bar{A}_{n}\right)^{\bar{\delta}}\right)=\lim _{n \rightarrow \infty} \mu\left(A_{n}^{\bar{\delta}}\right)
$$

which implies

$$
v(A)=\min \left(\mu\left(A^{\bar{\delta}}\right)+\varepsilon, 1\right)=\lim _{n \rightarrow \infty} \min \left(\mu\left(A_{n}^{\bar{\delta}}\right)+\varepsilon, 1\right)=\lim _{n \rightarrow \infty} v\left(A_{n}\right),
$$

so that $v$ is in fact a Dempster capacity.

On the other hand, Proposition [3.1 does not hold: take e.g. $X=[0,1], d=|\cdot|$ and $u=\mathbb{1}_{0}$, and any probability measure $\mu$. Then $u$ is clearly discontinuous. In particular for any compact set $K$ such that $\left.u\right|_{K}$ is continuous we must have $K \neq X$, so that $v(X \backslash K) \geq \varepsilon$ by definition of $v$. In particular Proposition 3.1 is not satisfied for $\tilde{\varepsilon}<\varepsilon$.

Examing [1] the problem seems to be here: in [1, Proof of Theorem 1] one has to check that $v$ is a capacity in the enlarged topology $\tau_{u}$, which makes u continuous. For $u=\mathbb{1}_{0}$, this means that necessarily $\tau_{u}$ contains $\{0\}$ and its complement. Consider now the $\tau_{u}$-closed sets $A_{n}=(0,1 / n]=(\{0\} \cup(1 / n, 1])^{c} \downarrow \emptyset$, for which $0=v(\emptyset)<$ 
$\delta=v\left(A_{n}\right)$, so $v$ is not a capacity wrt. $\tau_{u}$. In particular arguments of [1, Lemma 3] or [4, Lemma 2.2] do not apply and tightness of core $(v)$ is not satisfied.

\section{MAin Result}

From our discussion in Section 3 it seems that the assumptions on the capacity $v$ are not strong enough in order for a version of Lusin's theorem in the spirit of Proposition 3.1 to hold. In fact, the continuity property from above for Dempster capacities given by

$$
A_{n} \downarrow A, A_{n} \supseteq \bar{A}_{n+1} \forall n \in \mathbb{N} \Rightarrow \quad \lim _{n \rightarrow \infty} v\left(A_{n}\right)=v(A)
$$

is not sufficient, as it does not demand any continuity from above for the pathological case, where the sequence of $\left(A_{n}\right)_{n \in \mathbb{N}}$ satisfies $A_{n} \downarrow \emptyset$ and $A_{n} \neq \emptyset$ for all $n \in \mathbb{N}$. Contrary to this, continuity from above on the open sets seems to be crucial as stated in our main result, which we recall here for the the reader's convenience:

Theorem 1.1. Let $(X, d)$ be a compact metric space and let $v$ be a sub-additive capacity. Then the property

for all $\left(O_{n}\right)_{n \in \mathbb{N}}, O_{n}$ open, $O_{n} \downarrow O \quad \Rightarrow \quad \lim _{n \rightarrow \infty} v\left(O_{n}\right)=v(O)$

holds if and only if the property

for all $\varepsilon>0$ and for all Borel measurable functions $u: X \rightarrow \mathbb{R}$ there exists a compact set $K=K(u, \varepsilon) \subseteq X$, such that $v(X \backslash K) \leq \varepsilon$ and $\left.u\right|_{K}$ is continuous

holds.

Remark 4.1. Either from first principles or by checking the proof below, one concludes that the condition

$$
\text { for all }\left(O_{n}\right)_{n \in \mathbb{N}}, O_{n} \text { open, } O_{n} \downarrow O \quad \Rightarrow \quad \lim _{n \rightarrow \infty} v\left(O_{n}\right)=v(O)
$$

for a capacity $v$ is actually equivalent to

$$
\text { for all }\left(A_{n}\right)_{n \in \mathbb{N}}, A_{n} \downarrow A \Rightarrow \lim _{n \rightarrow \infty} v\left(A_{n}\right)=v(A),
$$

i.e. $v$ is continuous from above.

In Example 3.2 we have already observed that $v$ defined as the supremum of probability measures in a Prokhorov-ball around $\mu$ is not continuous from above on open sets. However, we can consider the following simple setting:

Example 4.2. Let $m \in \mathbb{N}$ and $\mu_{1}, \ldots, \mu_{m}$ be probability measures on $(X, d)$. Then $v$ defined via

$$
v(A):=\sup _{k=1, \ldots, m} \mu_{k}(A)
$$

for all sets $A \in \mathcal{B}$ is a sub-additive capacity, which is continuous from above on the open sets.

Proof. Fix a sequence of open sets $\left(O_{n}\right)_{n \in \mathbb{N}}$ such that $O_{n} \downarrow O$. Then as each $\mu_{k}$ is a proability measure, we have $\lim _{n \rightarrow \infty} \mu_{k}\left(O_{n}\right)-\mu_{k}(O)=0$ and thus also

$$
\lim _{n \rightarrow \infty} \sup _{k=1, \ldots, m} \mu_{k}\left(O_{n}\right)-\mu_{k}(O)=\sup _{k=1, \ldots, m} \lim _{n \rightarrow \infty} \mu_{k}\left(O_{n}\right)-\mu_{k}(O)=0 .
$$

The remaining properties of $v$ follow directly from the fact, that $\mu_{k}$ are probability measures. 
Lastly we remark that working on a compact metric space is not a severe restriction in this paper as the following result shows:

Lemma 4.3. Fix $\varepsilon>0$. If $v$ is a Choquet capacity, then there exists a compact set $K \subseteq X$ such that $v(X \backslash K) \leq \varepsilon$.

Proof. This follows from [4][Lemma 2.2], which states that the core of $v$ is tight.

\section{Proof of the main Result}

We start by showing that continuity from above on open sets implies Lusin's theorem for $v$. For this, we first introduce a stronger notion of regularity for capacities in comparison to [1]:

Definition 5.1. A set function $v: \mathcal{B} \rightarrow[0,1]$ is called regular, if the following holds: for all Borel sets $A$ and all $\varepsilon>0$ there exists an open set $O$ and a closed set $F$ such that $F \subseteq A \subseteq O$ and $v(O \backslash F) \leq \varepsilon$.

With this definition at hand, the next proposition follows naturally:

Proposition 5.2. Let $(X, d)$ be a compact metric space and let $v$ be a sub-additive capacity satisfying

$$
\text { for all }\left(O_{n}\right)_{n \in \mathbb{N}}, O_{n} \text { open, } O_{n} \downarrow O \quad \Rightarrow \quad \lim _{n \rightarrow \infty} v\left(O_{n}\right)=v(O) \text {. }
$$

Then $v$ is regular.

Proof. We define $\mathcal{M}$ as the collection of all Borel sets, which are regular for $v$, i.e. for all $\varepsilon>0$ there exists an open set $O$ and a closed set $F$ such that $F \subseteq A \subseteq O$ and $v(O \backslash F) \leq \varepsilon$. We show that $\mathcal{B} \subseteq \mathcal{M}$. Note that $\emptyset \in \mathcal{M}$, as it is clopen. Furthermore $\mathcal{M}$ is closed under complementation: indeed take a set Borel set $A$ and $\varepsilon>0$ such that there exists an open set $O$ and a closed set $F$ with $F \subseteq A \subseteq O$ and $v(O \backslash F) \leq \varepsilon$. Then we also have $O^{c} \subseteq A^{c} \subseteq F^{c}, F^{c}$ is open and $O^{c}$ is closed. Moreover, $v\left(F^{c} \backslash O^{c}\right)=v(O \backslash F) \leq \varepsilon$. In conclusion $A^{c} \in \mathcal{M}$.

Next we fix a collection $\left\{A_{n}\right\}_{n=1}^{\infty}$ of sets in $\mathcal{M}$. Our aim is to show that $\bigcup_{n=1}^{\infty} A_{n} \in$ $\mathcal{M}$. Let us fix $\varepsilon>0$, and for each $n \in \mathbb{N}$, let us take some open sets $O_{n}$ and closed sets $F_{n}$ such that $F_{n} \subseteq A_{n} \subseteq O_{n}$ and $v\left(O_{n} \backslash F_{n}\right) \leq 2^{-n-1} \varepsilon$. The we conclude that

$$
\bigcup_{n=1}^{N} F_{n} \subseteq \bigcup_{n=1}^{\infty} A_{n} \subseteq \bigcup_{n=1}^{\infty} O_{n}
$$

for any $N \in \mathbb{N}, \bigcup_{n=1}^{N} F_{n}$ is closed, $\bigcup_{n=1}^{\infty} O_{n}$ is open and thus by continuity from above on open sets (4)

$$
v\left(\bigcup_{n=1}^{\infty} O_{n} \backslash \bigcup_{n=1}^{N} F_{n}\right) \downarrow v\left(\bigcup_{n=1}^{\infty} O_{n} \backslash \bigcup_{n=1}^{\infty} F_{n}\right)
$$


for $N \rightarrow \infty$. On the other hand, by monotonicity (2) and sub-additivity (5) of $v$ we conclude that

$$
\begin{aligned}
v\left(\bigcup_{n=1}^{N} O_{n} \backslash \bigcup_{n=1}^{\infty} F_{n}\right) & \leq \sum_{n=1}^{N} v\left(O_{n} \backslash \bigcup_{n=1}^{\infty} F_{n}\right) \\
& \leq \sum_{n=1}^{N} v\left(O_{n} \backslash F_{n}\right) \\
& \leq \sum_{n=1}^{N} 2^{-n-1} \varepsilon .
\end{aligned}
$$

Lastly,

$$
\bigcup_{n=1}^{N} O_{n} \backslash \bigcup_{n=1}^{\infty} F_{n} \uparrow \bigcup_{n=1}^{\infty} O_{n} \backslash \bigcup_{n=1}^{\infty} F_{n}
$$

for $N \rightarrow \infty$, so that the above together with continuity from below (3) implies

$$
\begin{aligned}
v\left(\bigcup_{n=1}^{\infty} O_{n} \backslash \bigcup_{n=1}^{\infty} F_{n}\right) & =\lim _{N \rightarrow \infty} v\left(\bigcup_{n=1}^{N} O_{n} \backslash \bigcup_{n=1}^{\infty} F_{n}\right) \\
& \leq \lim _{N \rightarrow \infty} \sum_{n=1}^{N} 2^{-n-1} \varepsilon \leq \frac{\varepsilon}{2} .
\end{aligned}
$$

In particular, using (2), there exists $N \in \mathbb{N}$ such that

$$
v\left(\bigcup_{n=1}^{\infty} O_{n} \backslash \bigcup_{n=1}^{N} F_{n}\right) \leq \varepsilon .
$$

This shows that $\bigcup_{n \in \mathbb{N}} A_{n} \in \mathcal{M}$ and so $\mathcal{M}$ is a $\sigma$-algebra. We now show that it contains the closed sets. Indeed, take a closed set $F$ and recall that its open $\delta$-neighbourhood is given by

$$
F^{\delta}=\left\{x \in X: \inf _{y \in F} d(x, y)<\delta\right\} .
$$

Then $F^{\delta}$ is open for $\delta>0$ and as $F$ is closed we thus have $F^{\delta} \backslash F \downarrow \emptyset$, which implies by continuity from above on open sets (4) that

$$
v\left(F^{\delta} \backslash F\right) \downarrow v(\emptyset)=0 .
$$

This in particular implies that $\mathcal{B} \subseteq \mathcal{M}$, which concludes the proof.

We can now state the following version of Lusin's theorem for capacities:

Theorem 5.3. Let $(X, d)$ be a compact metric space and let $v$ be a sub-additive capacity satisfying

$$
O_{n} \text { open, } O_{n} \downarrow O \quad \Rightarrow \quad \lim _{n \rightarrow \infty} v\left(O_{n}\right)=v(O) .
$$

Let $u: X \rightarrow \mathbb{R}$ be a Borel measurable function. Given $\varepsilon>0$ there exists a compact set $K$ such that $v(X \backslash K) \leq \varepsilon$ and $\left.u\right|_{K}$ is continuous.

Given the regularity properties of $v$ established in Proposition 5.2 , this follows as in the classical case, see e.g. [2, Proof of Theorem 7.4.3]. 
Proof. First suppose that $u$ has the representation

$$
u=\sum_{n=1}^{\infty} a_{n} \mathbb{1}_{A_{n}}
$$

for real numbers $a_{n}$ and a countable partition of $X$ given by $\left\{A_{n}: n \in \mathbb{N}\right\}$. Fix $\varepsilon>0$. By Proposition 5.2 we can find open sets $O_{n}$ and closed sets $F_{n}$ such that $F_{n} \subseteq A_{n} \subseteq O_{n}$ and $v\left(O_{n} \backslash F_{n}\right) \leq 2^{-n-2} \varepsilon$. Thus in particular

$$
X=\bigcup_{n \in \mathbb{N}} A_{n} \subseteq \bigcup_{n \in \mathbb{N}} O_{n} \subseteq X,
$$

so that $X=\bigcup_{n \in \mathbb{N}} O_{n}$. As in the proof of Proposition 5.2 there exists $N \in \mathbb{N}$ such that

$$
v\left(\bigcup_{n=1}^{\infty} O_{n} \backslash \bigcup_{n=1}^{N} F_{n}\right) \leq \varepsilon / 2
$$

and so

$$
\begin{aligned}
v\left(\bigcup_{n=N+1}^{\infty} A_{n}\right) & =v\left(X \backslash \bigcup_{n=1}^{N} A_{n}\right) \\
& \leq v\left(X \backslash \bigcup_{n=1}^{N} F_{n}\right) \\
& =v\left(\bigcup_{n=1}^{\infty} O_{n} \backslash \bigcup_{n=1}^{N} F_{n}\right) \\
& \leq \varepsilon / 2,
\end{aligned}
$$

where we used the fact that $\left\{A_{n}: n \in \mathbb{N}\right\}$ is a partition in the first equality and $\bigcup F_{n} \subseteq \bigcup A_{n}$ for the first inequality. Take the compact set $K=\bigcup_{n=1}^{N} F_{n}$. Again using the fact that $\left\{A_{n}: n \in \mathbb{N}\right\}$ is a partition and $F_{n} \subseteq A_{n}$, we conclude that $F_{n}$ are disjoint. Note that $u$ is constant on each $F_{n}$, so it is clearly continuous on $K$. Furthermore using sub-additivity (5) we have

$$
\begin{aligned}
v(X \backslash K) & \leq v\left(\bigcup_{n=1}^{N} A_{n} \backslash \bigcup_{n=1}^{N} F_{n}\right)+v\left(\bigcup_{n=N+1}^{\infty} A_{n}\right) \\
& \leq \sum_{n=1}^{N} v\left(O_{n} \backslash F_{n}\right)+\varepsilon / 2 \\
& \leq \varepsilon / 2+\varepsilon / 2=\varepsilon .
\end{aligned}
$$

Now let $u$ be an arbitrary Borel measurable function. Then $u$ is the uniform limit of a sequence $\left(u_{n}\right)_{n \in \mathbb{N}}$ of functions, each of which is Borel measurable and has only countably many values (for example, $u_{n}$ might be defined by letting $u_{n}(x)$ be $k / n$, where $k$ is the integer that satisfies $k / n \leq u(x)<(k+1) / n)$. The above then implies that for each $n \in \mathbb{N}$ there is a compact subset $K_{n}$ of $X$ such that $v\left(X \backslash K_{n}\right)<2^{-n} \varepsilon$ and such that the restriction of $u_{n}$ to $K_{n}$ is continuous. Let $K=\bigcap_{n=1}^{\infty} K_{n}$. Then $K$ is a compact subset of $X$,

$$
v(X \backslash K) \leq \sum_{n=1}^{\infty} \mu\left(X \backslash K_{n}\right) \leq \sum_{n=1}^{\infty} 2^{-n} \varepsilon=\varepsilon
$$


and $u$ is continuous on $K$ as the uniform limit of the functions $u_{n}$, which are continuous on $K$. This concludes the proof.

We now complete the proof via the following proposition:

Proposition 5.4. Assume that $(X, d)$ is compact metric space and $v$ is a subadditive capacity satisfying the property

for all $\varepsilon>0$ and for all Borel measurable functions $u: X \rightarrow \mathbb{R}$ there exists a compact set $K=K(u, \varepsilon) \subseteq X$, such that $v(X \backslash K) \leq \varepsilon$ and $\left.u\right|_{K}$ is continuous.

Then for all $\left(O_{n}\right)_{n \in \mathbb{N}}, O_{n}$ open, $O_{n} \downarrow O \quad \Rightarrow \quad \lim _{n \rightarrow \infty} v\left(O_{n}\right)=v(O)$.

Proof. Fix a sequence $\left(O_{n}\right)_{n \in \mathbb{N}}$ of open sets such that $O_{n} \downarrow O$. Take $\varepsilon>0$. Our aim is to show that $v\left(O_{n}\right)-v(O) \leq \varepsilon$ for $n$ large enough. Note that sub-additivity of $v$ implies that for Borel sets $A \subseteq B$ we have

$$
v(B)-v(A) \leq v(B \backslash A) .
$$

In particular we can assume that $v(X \backslash O)>0$ : indeed, if this is not the case then (3) implies that

$$
0 \leq v(X)-v(O) \leq v(X \backslash O)=0,
$$

so that $v(O)=v(X)=1$. Thus we conclude $v\left(O_{n}\right)=1$ for all $n \in \mathbb{N}$ by monotonicity of $v$ and $O_{n} \supseteq O$, which means $\inf _{n \in \mathbb{N}} v\left(O_{n}\right)-v(O)=0$, in particular $v\left(O_{n}\right)-v(O) \leq \varepsilon$ for $n$ large enough.

To simplify notation we now define

$$
\tilde{v}(A):=\frac{v(A \backslash O)}{v(X \backslash O)}
$$

for Borel sets $A \subseteq X$. We note that $\tilde{v}(A)=\tilde{v}(A \backslash O)$ and that $\tilde{v}$ is again a sub-additive capacity. Furthermore, sub-additivity of $v$ implies that

$$
\inf _{n \in \mathbb{N}} \frac{v\left(O_{n}\right)-v(O)}{v(X \backslash O)} \leq \inf _{n \in \mathbb{N}} \frac{v\left(O_{n} \backslash O\right)}{v(X \backslash O)}=\inf _{n \in \mathbb{N}} \tilde{v}\left(O_{n} \backslash O\right),
$$

so it is sufficient to show that

$$
\tilde{v}\left(O_{n} \backslash O\right) \leq \frac{\varepsilon}{v(X \backslash O)}=: \tilde{\varepsilon}
$$

for $n$ large enough. We now define the sequence of functions $\left(u_{n}\right)_{n \in \mathbb{N}}$ via

$$
u_{n}:= \begin{cases}1 & \text { if } x \in O_{n} \backslash O \\ 0 & \text { otherwise. }\end{cases}
$$

Then for each $n \in \mathbb{N}$ we can apply Lusin's theorem to $\tilde{v}$ in order to obtain a compact set $K_{n} \subseteq X$ such that $\tilde{v}\left(X \backslash K_{n}\right) \leq 2^{-n} \tilde{\varepsilon}$ and $\left.u_{n}\right|_{K_{n}}$ is continuous. Then necessarily

$$
\inf \left\{d(x, y): x \in K_{n} \cap\left(O_{n} \backslash O\right), y \in K_{n} \cap\left(O_{n} \backslash O\right)^{c}\right\}>0 .
$$

Indeed otherwise there exists $x \in K_{n}$ such that $x \in \overline{K_{n} \cap\left(O_{n} \backslash O\right)} \cap \overline{K_{n} \cap\left(O_{n} \backslash O\right)^{c}}$, which contradicts continuity of $\left.u_{n}\right|_{K_{n}}$. From (4) we then conclude that $K_{n} \cap\left(O_{n} \backslash O\right)$ is compact. Now we define a new sequence $\left(\tilde{K}_{n}\right)_{n \in \mathbb{N}}$ of compact sets given by

$$
\tilde{K}_{n}=\bigcap_{l=1}^{n} K_{l} \subseteq K_{n}
$$


for all $n \in \mathbb{N}$. Then clearly $\tilde{K}_{n}$ is decreasing, $\tilde{K}_{n} \cap\left(O_{n} \backslash O\right)$ is compact as a finite intersection of compact sets and

$$
\begin{aligned}
\tilde{v}\left(\left(O_{n} \backslash O\right) \backslash \tilde{K}_{n}\right) & \leq \tilde{v}\left(X \backslash \tilde{K}_{n}\right) \\
& =\tilde{v}\left(X \backslash \bigcap_{l=1}^{n} K_{l}\right) \\
& =\tilde{v}\left(\bigcup_{l=1}^{n} X \backslash K_{l}\right) \\
& \leq \sum_{l=1}^{n} \tilde{v}\left(X \backslash K_{l}\right) \\
& \leq \sum_{l=1}^{n} 2^{-l} \tilde{\varepsilon} \\
& \leq \tilde{\varepsilon}
\end{aligned}
$$

for all $n \in \mathbb{N}$. Furthermore

$$
\tilde{K}_{n} \cap\left(O_{n} \backslash O\right) \subseteq O_{n} \backslash O \downarrow \emptyset
$$

as $n \rightarrow \infty$, which directly implies

$$
\tilde{K}_{n} \cap\left(O_{n} \backslash O\right) \downarrow \emptyset
$$

as $n \rightarrow \infty$. As $\tilde{K}_{n} \cap\left(O_{n} \backslash O\right)$ is compact and decreasing, there exists $n_{0} \in \mathbb{N}$ such that

$$
\tilde{K}_{n} \cap\left(O_{n} \backslash O\right)=\emptyset
$$

for all $n \geq n_{0}$. In particular

$$
\tilde{v}\left(\tilde{K}_{n} \cap\left(O_{n} \backslash O\right)\right)=\tilde{v}(\emptyset)=0
$$

for all $n \geq n_{0}$. In conclusion, using sub-additivity of $\tilde{v}$, (5) and (6),

$$
\begin{aligned}
\tilde{v}\left(O_{n} \backslash O\right) & \leq \tilde{v}\left(\tilde{K}_{n} \cap\left(O_{n} \backslash O\right)\right)+\tilde{v}\left(\left(O_{n} \backslash O\right) \backslash \tilde{K}_{n}\right) \\
& \leq \tilde{\varepsilon}
\end{aligned}
$$

for $n \geq n_{0}$. This concludes the proof.

\section{REFERENCES}

[1] Adriana Castaldo and Massimo Marinacci. A Lusin theorem for a class of Choquet capacities. Statist. Papers, 43(1):137, 2002.

[2] D. Cohn. Measure theory. Birkhäuser, 1980.

[3] Wu Congxin and Ha Minghu. On the regularity of the fuzzy measure on metric fuzzy measure spaces. Fuzzy Sets and Systems, 66(3):373-379, 1994.

[4] Peter J Huber and Volker Strassen. Minimax tests and the Neyman-Pearson lemma for capacities. Ann. Statist., pages 251-263, 1973.

[5] Jun Li and Masami Yasuda. Lusin's theorem on fuzzy measure spaces. Fuzzy Sets and Systems, 146(1):121-133, 2004.

[6] Nikolai Lusin. Sur les propriétés des fonctions mesurables. C. R. Math. Acad. Sci. Paris, 154(25):1688-1690, 1912.

[7] Zhaojun Zong, Feng Hu, and Xiaoxin Tian. Egoroff's theorem and Lusin's Theorem for Capacities in the Framework of $g$-Expectation. Math. Probl. Eng., 2020, 2020. 
JoHANNES WIESEL

Columbia University, Department of Statistics

1255 Amsterdam Avenue

New York, NY 10027, USA

Email address: johannes.wiesel@columbia.edu 